\section{Correlations in intronless DNA}

SIR - Peng et al. ${ }^{1}$ have reported their finding of long-range correlations in DNA sequences, analysed as purine/ pyrimidine 'random' walks. In addition, they suggested that it is possible to distinguish intronless DNA sequences from sequences containing introns using the value of the exponent of a power law calculated on these sequences. They supposed that intronless sequences yield exponents of approximately 0.5 and those containing introns yield exponents greater than 0.5. Nee in Scientific Correspondence $^{2}$ has put forward a different framework for interpreting those results in terms of the already known mosaic structure of genomes.

We tested the algorithm of Peng et al. on the 80 largest intronless putative coding regions of yeast chromosome III (ref. 3) with lengths ranging from 1,000 to 6,000 nucleotides. On one strand, 39 such coding regions have been identified; 7 of these open reading frames
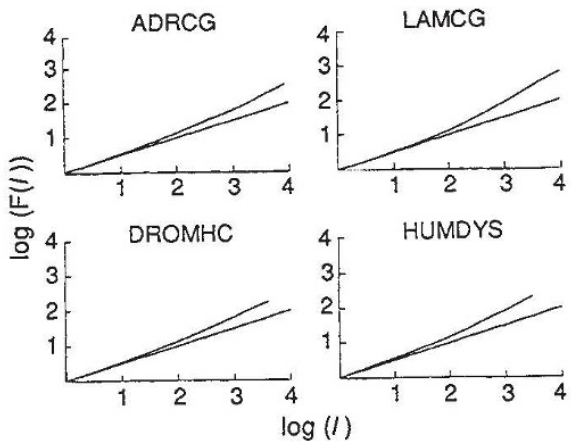

The two frames on the left are plots of log $(F(I))$ against log (I) for intron-containing sequences reported in ref. 1 . The two frames on the right correspond to intronless sequences reported in the same paper. In each frame the continuous curve is obtained if $F(I)$ is calculated as an average over the entire sequence. A straight line of slope $\alpha=0.5$ is included in each plot for comparison.

and those containing introns (see figure). Moreover, in intronless sequences the reported slope of 0.5 appears to be an artefact of the min-max partitioning. The slope is much higher when $F(l)$ is computed over the entire sequence (column 2 of the table). Finally, when the fluctuation is computed as an average over the entire sequence, the slope deviates from 0.5 as soon as the window size exceeds 10-50 nucleotides (see table). This is true for all but one of the 10 intronless sequences reported in ref. 1 . The table shows the increase of the slope $\alpha$ with the window size; values appreciably higher than 0.5 are obtained for a window size of 100 , representing less than $5 \%$ of the length of the smallest intronless se-

yielded exponents between 0.41 and $0.47 ; 11$ had exponents between 0.47 and 0.53 ; and 21 had exponents between 0.54 and 0.73 . On the complementary strand, 41 such coding regions have been identified. Of these, 6 yielded exponents between 0.38 and $0.47 ; 12$ yielded exponents between 0.47 and 0.53 ; and 23 had exponents between 0.54 and 0.68 .

Failing to discern any clear correlation, we decided to verify the algorithm for the same sequences whose exponents were reported by Peng et al. ${ }^{1}$. The existence of a power law such as $F(l) \sim$ $1^{\alpha}$ implies that the plot of $\log (F(l))$ against $\log (l)$ is a straight line, the slope of which is $\alpha$. We found that, in most cases, the plot of $\log (F(l))$ against $\log$ $(l)$, where $F(l)$ is calculated as an average over the entire sequence, is not a straight line. Nonlinear curves were obtained both for intronless sequences

\section{Antibodies from libraries}

SIR - We have predicted ${ }^{1-3}$ that combinatorial antibody technology will eventually become the method of choice for the generation of monoclonal antibodies and we stick by that prediction despite the reservations expressed in the Scientific Correspondence by Gherardi and Milstein ${ }^{4}$. In particular, we believe the potential to derive high-affinity human antibody fragments from combinatorial libraries has been clearly demonstrated ${ }^{5-8}$. Most recently, we have shown recombinant Fab fragments against the HIV-1 surface glycoprotein gp120 or against the respiratory syncytial virus F-glycoprotein to be highly effective in neutralizing the respective viruses ${ }^{9}$. Nevertheless, Gherardi and Milstein raise interesting issues about the scrambling of heavy $(\mathrm{H})$ and light (L) chains inherent in the random combinatorial approach and the likelihood of regenerating the in vivo $\mathrm{H}-\mathrm{L}$ combination ('original antibody' in the nomenclature of Gherardi and Milstein).

Do $\mathrm{H}$ and $\mathrm{L}$ chains find their original partners in random combinatorial libraries? It has been concluded previously ${ }^{10}$ on statistical grounds that they cannot unless one has very large libraries, thus Gherardi and Milstein argue for very complex combinatorial libraries $\left(>10^{8}\right)$. However, where it has been looked at, the representation of functional $\mathrm{H}$ and $\mathrm{L}$ chains in combinatorial immunoglobulin $\mathrm{G}$ libraries prepared from immunized animals has been far higher than these theoretical statistical arguments seem to allow. For instance, a study on mice infected with influenza $A$ virus ${ }^{11}$ found a functional $\mathrm{H}$ chain at a frequency of 1 in 50 in a combinatorial library and a functional $\mathrm{L}$ chain at a frequency of 1 in 275 . The expected frequency of the combination (anti-haemagglutinin) is therefore only 1 in 13,750 and would be present in multiple copies in a library as small as $10^{5}$. These frequencies are determined by hybridization experiments, which may not distinguish somatic variants of given $\mathrm{H}$ and $\mathrm{L}$ chains and therefore do not unambiguously describe original $\mathrm{H}-\mathrm{L}$ combinations according to a strict definition of chains arising from the same B cell. But they do indicate that the diversity of $\mathrm{IgG}$ libraries from immunized animals can be restricted and theoretical statistical arguments are fraught with difficulties. In this context, bigger libraries are not necessarily better libraries: library quality in terms of functional binders is dependent on the source of mRNA.

Gherardi and Milstein believe that $\mathrm{H}$ and $L$ chains do not find their original NATURE · VOL 359 - 29 OCTOBER 1992 